\title{
Differential regulation of monocarboxylate transporter 8 expression in thyroid cancer and hyperthyroidism
}

\author{
Julia Badziong', Saskia Ting' ${ }^{2}$, Sarah Synoracki², Vera Tiedje', Klaudia Brix ${ }^{3}$, \\ Georg Brabant ${ }^{4}$, Lars Christian Moeller ${ }^{1}$, Kurt Werner Schmid ${ }^{2}$, Dagmar Fuhrer ${ }^{1}$ \\ and Denise Zwanziger ${ }^{1}$
}

1'Department of Endocrinology and Metabolism and Division of Laboratory Research, University of Duisburg-Essen, Essen, Germany, ${ }^{2}$ University Hospital Essen, Institute of Pathology, Essen, Germany, ${ }^{3}$ Department of Life Sciences and Chemistry, Jacobs University Bremen, Bremen, Germany, and ${ }^{4}$ University Hospital Schleswig-Holstein, Experimental and Clinical Endocrinology, Lübeck, Germany
Correspondence should be addressed to D Zwanziger Email

denise.zwanziger@uk-essen.de

\begin{abstract}
Objective: Thyroid hormone (TH) transporters are expressed in thyrocytes and most play a role in TH release. We asked whether expression of the monocarboxylate transporter 8 (MCT8) and the L-type amino acid transporters LAT2 and LAT4 is changed with thyrocyte dedifferentiation and in hyperfunctioning thyroid tissues.

Design and methods: Protein expression and localization of transporters was determined by immunohistochemistry in human thyroid specimen including normal thyroid tissue (NT, $n=19$ ), follicular adenoma (FA, $n=44)$, follicular thyroid carcinoma (FTC, $n=45$ ), papillary thyroid carcinoma (PTC, $n=40$ ), anaplastic thyroid carcinoma (ATC, $n=40)$ and Graves' disease $(G D, n=50)$ by calculating the 'hybrid' $(H)$ score. Regulation of transporter expression was investigated in the rat follicular thyroid cell line PCCL3 under basal and thyroid stimulating hormone (TSH) conditions.

Results: MCT8 and LAT4 were localized at the plasma membrane, while LAT2 transporter showed cytoplasmic localization. MCT8 expression was downregulated in benign and malignant thyroid tumours as compared to NT. In contrast, significant upregulation of MCT8, LAT2 and LAT4 was found in GD. Furthermore, a stronger expression of MCT8 was demonstrated in PCCL3 cells after TSH stimulation.

Conclusions: Downregulation of MCT8 in thyroid cancers qualifies MCT8 as a marker of thyroid differentiation. The more variable expression of LATs in distinct thyroid malignancies may be linked with other transporter properties relevant to altered metabolism in cancer cells, i.e. amino acid transport. Consistent upregulation of MCT8 in GD is in line with increased TH release in hyperthyroidism, an assumption supported by our in vitro results showing TSH-dependent upregulation of MCT8.
\end{abstract}

European Journal of

Endocrinology

(2017) 177, 243-250

\section{Introduction}

Thyroid hormones (TH) are essential for cell differentiation, growth and metabolism (1). TH influx and efflux in tissues is regulated by TH transporters. So far, the most specific $\mathrm{TH}$ transporter is the monocarboxylate transporter 8 (MCT8) $(2,3)$ which is expressed in brain, heart, kidney, liver, skeletal muscle, placenta, testis and the basolateral plasma membrane of thyroid epithelial
두 2017 European Society of Endocrinology Printed in Great Britain cells consistent with its role in thyroxine (T4) export from thyroid follicles $(4,5,6)$. The preferred substrate of MCT8 is 3,3',5-triiodothyronine (T3), but pharmacological studies showed that other THs such as T4, reverse T3 (rT3) and 3,5-diiodo-L-thyronine (T2) are transported as well (7). Secondary TH transporters, i.e. the large neutral amino acid transporters LAT2 and LAT4 facilitate in and

Published by Bioscientifica Ltd. 
efflux of TH and other substrates $(8,9,10)$. Thus, while the preferred TH substrate of LAT2 is 3,3'-T2 (4), LAT2 also transports large neutral amino acids, amino acid-related compounds and small amino acids (11). LAT2 is expressed in many tissues including the thyroid gland (4). LAT4 is a sodium, chloride and $\mathrm{pH}$ independent transporter (12) and is detected in kidney, placenta, peripheral blood leukocytes and spleen (12). Recent studies suggest that LAT4 is not involved in T3 and T4 transport but facilitates T2 efflux (10). Thus, known TH transporters differ in terms of substrate preferences, direction of TH transport (efflux and influx) and tissue expression pattern.

$\mathrm{TH}$ transporter expression in the thyroid gland was first demonstrated by Di Cosmo et al. in an elegant Mct8 mouse knock-out study (3). Mct8 expression was found at the basolateral membrane of thyrocytes (3) strongly suggesting that Mct8 plays a role in TH release. Subsequent studies in mice have shown that Lat2 is also expressed in the thyroid gland (3) with a cytoplasmic localization.

Whether alterations of thyroid differentiation or thyroid function may have an impact on $\mathrm{TH}$ transporter expression in the thyroid is currently unknown. In this study we asked whether expression and/or localization of MCT8 and secondary TH transporters differ between benign and malignant thyroid tumours and in hyperfunctioning vs normal thyroid tissues (NTs). Based on our findings we suggest that MCT8 is a suitable marker for thyroid differentiation and that MCT8 upregulation occurs in hyperfunctional thyroid tissue consistent with its role in TH release and with TSH-dependent TH synthesis and release.

\section{Subjects and methods}

\section{Thyroid samples}

Thyroid tissue samples from 238 patients were investigated. Histological classification of tissue specimen according to WHO criteria was obtained by certified pathologists. TH transporter expression was investigated on paraffin-embedded tissues of 19 NTs, 44 follicular adenomas (FA), 45 follicular thyroid carcinomas (FTC), 40 papillary thyroid carcinomas (PTC), 40 anaplastic thyroid carcinomas (ATC) and 50 Graves' disease (GD) thyroid specimen.

\section{Immunohistochemistry}

For immunohistochemical analysis the following antibodies were used: anti-MCT8/SLC16A2 (1:150,
\#HPA003353, Atlas Antibodies, Bromma, Sweden), antiLAT2 (1:200, \#0142-10, immunoGlobe, Himmelstadt, Germany) and anti-LAT4 (1:10, \#HPA021564, Atlas Antibodies). All tissue sections were deparaffinized and rehydrated through graded series of alcohols (70\%-/96\%-/ $100 \% \mathrm{v} / \mathrm{v}$ ethanol, Sigma-Aldrich). Pretreatment was performed for $20 \mathrm{~min}$ in citrate buffer $(\mathrm{pH} 6.0)$ at $95^{\circ} \mathrm{C}$. Tissue sections were blocked in an aqueous hydrogen peroxide solution $\left(3 \% \mathrm{v} / \mathrm{v} \mathrm{H}_{2} \mathrm{O}_{2}\right.$, Carl Roth, Karlsruhe, Germany) Primary antibodies were incubated for $30 \mathrm{~min}$ at RT. Immunoreactivity was demonstrated using a classical polymer system (Zytomed, Berlin, Germany). Cell nuclei were stained with haematoxylin (1:8; Dako) for $5 \mathrm{~min}$ and sections were mounted in Entellan (Merck). All steps were performed in a semi-automated fashion using the Dako Autostainer (Dako). Paraffin-embedded human kidney tissue sections were used as positive control. Negative controls (no primary antibody) were included in the experimental set-up. The Olympus BX51 upright microscope (Olympus) was used for light microscopy. Tumour staining intensities were evaluated by calculating the 'hybrid' (H) scores as previously described (13).

\section{Cell culture}

For cell culture experiments the rat follicular thyroid cell line PCCL3 (14) was used (kindly provided by Pilar Santisteban, Instituto de Investigaciones Biomédicas 'Alberto Sols' CSIC-UAM, Madrid, Spain). Cells were cultured in Ham's F12 (Invitrogen) medium with 5\% w/v fetal bovine serum (FBS, Invitrogen), $5 \mu \mathrm{g} / \mathrm{mL}$ transferrin, $10 \mu \mathrm{g} / \mathrm{mL}$ insulin, $10 \mathrm{ng} / \mathrm{mL}$ somatostatin, $1 \mathrm{U} / \mathrm{mL}$ thyroid stimulating hormone (TSH) and $10 \mathrm{nM}$ hydrocortisone (all from Sigma-Aldrich). PCCL3 cells were used between passages 5 and 15 . Cells were grown at $37^{\circ} \mathrm{C}$ and $5 \% \mathrm{CO}_{2}$. PCCL3 cell line was re-authenticated by mRNA expression profile of TH markers (Tg, Tpo, Nis and Thox1).

\section{Three-dimensional culturing of PCCL3 cells}

For cultivation of PCCL3 cells in a hanging drop culture, Methocel medium containing Ham's F12 and methylcellulose powder (Sigma-Aldrich) was prepared. The powder $(0.012 \mathrm{~g} / \mathrm{mL})$ was dissolved in warm medium $\left(50^{\circ} \mathrm{C}\right)$ and swirled for three days at RT. Subsequently $2 \mathrm{~mL}$ of the Methocel medium were mixed with $8 \mathrm{~mL}$ of normal PCCL3 medium and 400000 PCCL3 cells. Then droplets with a total volume of $25 \mu \mathrm{L}$ were placed on a cell culture dish and incubated upside down for $48-72 \mathrm{~h}$ at $37^{\circ} \mathrm{C}$ and $5 \%$ 
$\mathrm{CO}_{2}$. The formed spheroids in the droplets were harvested and centrifuged for $7 \mathrm{~min}$ at $200 \mathrm{~g}$. After supernatant was removed, spheroids were suspended in $200 \mu \mathrm{L}$ of warm Histogel (Thermo Scientific) and placed in a Cryomold on ice. After curing, cells were fixed with Histofix (Carl Roth) for $24 \mathrm{~h}$. Subsequently cells were paraffin-embedded and analysed by immunohistochemistry.

\section{Stimulation of PCCL3 cells}

For stimulation experiments, PCCL3 cells (50000 cells/mL) were seeded in 6-well plates and cultured for three days until $70-80 \%$ of confluence. Prior to stimulation cells were starved for $72 \mathrm{~h}$ with medium containing Ham's F12 without serum and hormones (starvation medium). Then cells were incubated in presence of $10 \mathrm{U} / \mathrm{mL} \mathrm{TSH}$ (Sigma-Aldrich) for $8 \mathrm{~h}$.

\section{Immunoblot}

The following antibodies were used: anti-MCT8 (1:1000, \#HPA003353, Atlas Antibodies), anti-LAT2 (1:200, \#014210, immunoGlobe), anti-LAT4 (1:10, \#HPA021564, Atlas Antibodies), anti-GAPDH (1:6000, \#ACT001P, Acris Antibodies, Herford, Germany), anti-rabbit IgG HRPlinked antibody (1:2000, \#7074, Cell Signaling) and anti-rabbit IgG DyLight 488 (1:5000, \#35553, Thermo Fisher). Whole protein lysates were extracted by radioimmunoprecipitation assay lysis (RIPA)-buffer $(150 \mathrm{mM}$ sodiumchloride, $50 \mathrm{mM}$ Tris hydrochloride, $1 \% \mathrm{v} / \mathrm{v}$ Tergitol (NP-40), $0.5 \% \mathrm{w} / \mathrm{v}$ sodiumdeoxycholate, $0.1 \% \mathrm{w} / \mathrm{v}$ sodium dodecyl sulphate, $2 \mathrm{mM}$ ethylenediaminetetraacetic acid and $50 \mathrm{mM}$ sodium fluoride, all from Sigma-Aldrich). In addition, a phosphatase and protease inhibitor cocktail (both from Roche) were added to the buffer. Lysed cells were incubated on ice for $20 \mathrm{~min}$ and then centrifuged for $20 \mathrm{~min}$ at $4^{\circ} \mathrm{C}$ and $13300 \mathrm{rpm}$. Extracted proteins were quantified by BCA protein assay (Pierce/Thermo Scientific). Aliquots of proteins $(20 \mu \mathrm{g})$ were separated on an $8 \%$ SDS-gel, blotted onto a polyvinylidene difluoride (PVDF, BioRad) membrane using the wet-blot technique at $4^{\circ} \mathrm{C}$ overnight (BioRad). Unspecific binding sites were blocked with $5 \% \mathrm{w} / \mathrm{v}$ non-fat milk (Carl Roth) or 5\% $\mathrm{w} / \mathrm{v}$ bovine serum albumin (BSA, Sigma-Aldrich) for $1 \mathrm{~h}$ at RT. Primary antibodies were incubated overnight at $4^{\circ} \mathrm{C}$ in $5 \% \mathrm{w} / \mathrm{v} \mathrm{BSA} / \mathrm{T}-\mathrm{BST}$. Incubation of the secondary antibody was performed for $2 \mathrm{~h}$ at RT in $2.5 \% \mathrm{w} / \mathrm{v}$ nonfat milk/T-BST. The visualization of the proteins was done by luminescence using the Immun-Star WesternC Kit
(BioRad) or by fluorescence dependent on the secondary antibody. Differences in protein expression levels were quantified by densitometry using the ImageLab Software (BioRad). Relative values of the loading control GAPDH as well as MCT8, LAT2 or LAT4 were calculated. The target protein values were divided by the calculated relative values of the respective control. The adjusted values were used to calculate the geometric mean of the controls and target protein followed by calculation of the percent of protein level alteration.

\section{Immunofluorescence}

PCCL3 cells ( 50000 cells $/ \mathrm{mL}$ ) were seeded on cover slides and incubated at $5 \% \mathrm{CO}_{2}$ and $37^{\circ} \mathrm{C}$ for $48 \mathrm{~h}$. Cells were washed twice with phosphate buffer saline (PBS, Invitrogen) for $5 \mathrm{~min}$ and then fixed with $4 \%$ paraformaldehyde (PFA, Sigma-Aldrich) for $15 \mathrm{~min}$ at RT. PFA was aspirated, cells were washed three times with PBS and were permeabilized with $0.1 \% \mathrm{v} / \mathrm{v}$ Triton X-100 (Sigma-Aldrich) in PBS for 10 min at RT. Blocking was performed by using 3\% w/v BSA (Sigma-Aldrich) in PBS for $1 \mathrm{~h}$ at RT. Cells were washed three times with $0.1 \% \mathrm{w} / \mathrm{v}$ BSA/PBS and a specific primary antibody against MCT8 (1:1000, \#HPA003353, Atlas Antibodies) was diluted in $0.1 \% \mathrm{w} / \mathrm{v}$ BSA/PBS. After incubation with the primary antibody $\left(4^{\circ} \mathrm{C}\right.$, overnight) cells were washed six times with $0.1 \% \mathrm{w} / \mathrm{v}$ BSA/PBS. Then, cells were incubated with the secondary antibody AlexaFluor 488 goat anti-rabbit (1:250, Invitrogen) for $1 \mathrm{~h}$ at RT in $0.1 \%$ $\mathrm{w} / \mathrm{v}$ BSA/PBS. Cells were washed six times with $0.1 \% \mathrm{w} / \mathrm{v}$ BSA/PBS for $5 \mathrm{~min}$ and detection of the cytoskeleton was performed by incubation with Phalloidin 555 (1:60, Invitrogen) in $0.1 \% \mathrm{w} / \mathrm{v}$ BSA/PBS for $20 \mathrm{~min}$ at RT. Visualizing of the nucleus was performed with Draq5 (1:500, BioStatus, Loughborough, UK) for $1 \mathrm{~h}$ at RT. Cover slides were embedded in ImmuMount (Thermo Scientific) and viewed with an LSM 510 Meta confocal microscope (Carl Zeiss Jena GmbH).

\section{Statistical analysis}

Results of the H-Score analysis are shown as mean \pm standard error of the mean (S.E.M.). Analysis was performed by one-way analysis of variance (ANOVA) with Bonferroni's multiple comparison post-hoc test using GraphPad Prism 5 software (GraphPad). Expression in thyroid carcinoma samples was compared to follicular adenoma and NTs. Expression in GD tissues was compared to NTs. For 
immunoblot analysis Student's $t$-test was performed. Differences were considered statistically significant if ${ }^{*} P<0.05,{ }^{* *} P<0.01$ and ${ }^{* *} P<0.001$.

\section{Results}

\section{TH transporter expression differs significantly between benign and malignant thyroid tumours}

TH transporter expression was investigated in FA, FTC, PTC, ATC and NTs respectively. Plasma membrane staining was found for MCT8 and LAT4 while LAT2 showed mainly cytoplasmic localization (Fig. 1).

The mean H-Score of MCT8, LAT2 and LAT4 differed between thyroid tumour entities. MCT8 was significantly downregulated in malignant thyroid tumours as compared to NT. LAT2 was significantly upregulated in PTC as compared to NT. LAT4 was significantly downregulated in ATC as compared to NT, whereas LAT4 staining in other thyroid tumours revealed a more heterogeneous expression pattern (Fig. 1 and Table 1).

\section{TH transporter expression is increased in hyperfunctional Graves' disease tissues}

To address whether expression of $\mathrm{TH}$ transporters is altered in hyperfunctional thyroid tissues, immunohistochemical analysis of MCT8, LAT2 and LAT4 was performed in GD tissue and was compared to NTs. Significantly enhanced staining was observed for MCT8, LAT2 and LAT4 in Graves' tissues (Table 2). Membrane localization was confirmed for MCT8 and LAT4 while LAT2 was localized in the cytoplasm (Fig. 2).

\section{MCT8 expression in rat thyroid cells is upregulated by TSH stimulation}

To investigate which pathway may contribute to $\mathrm{TH}$ transporter expression, we performed in vitro experiments in rat thyroid PCCL3 cells and focused on MCT8, LAT2 and LAT4 because these TH transporters showed distinct expression patterns for hyperfunctional thyroid tissues and/or malignant thyroid tumours. MCT8 expression and localization was determined in PCCL3 cells using

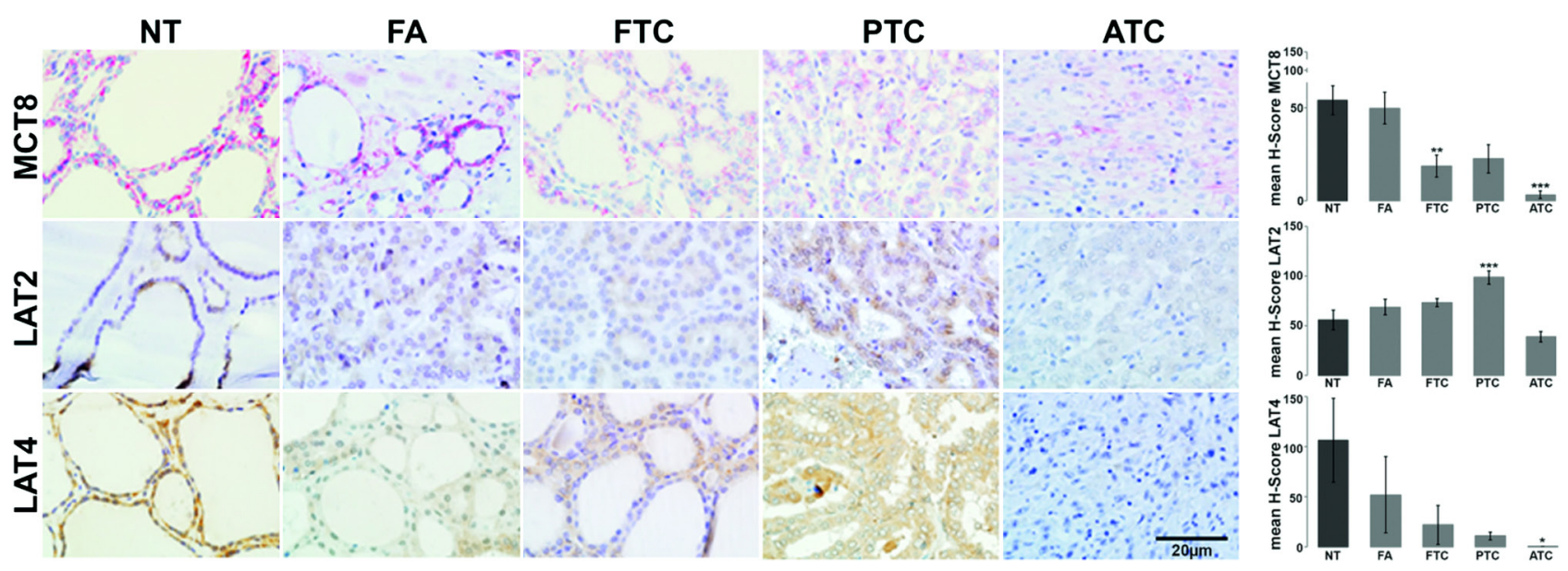

Figure 1

TH transporter expression differs significantly between benign and malignant thyroid tissue. Immunohistochemical analysis of TH transporters in normal thyroid tissue (NT), follicular adenoma (FA), follicular thyroid carcinoma (FTC), papillary thyroid carcinoma (PTC) and anaplastic thyroid carcinoma (ATC). Monocarboxylate transporter 8 (MCT8) is localized at the basolateral plasma membrane. Downregulation of MCT8 is found in thyroid carcinoma as compared to NT. L-type amino acid transporter type 2 (LAT2) is localized in the cytoplasm. LAT2 is significantly upregulated in PTC as compared to NT. LAT4 is localized in the plasma membrane and LAT4 downregulation was found in ATC compared to NT. Immunostaining in thyroid tumours was normalized to NT. Data are represented as mean H-Scores, mean \pm S.E.M. NT: $n=5$ (LAT4) or $n=19$ (MCT8, LAT2). FA: $n=5$ (LAT4) or $n=19$ (LAT2) and $n=44$ (MCT8). FTC: $n=5$ (LAT4) or $n=45$ (LAT2, MCT8). PTC: $n=5$ (LAT4) or $n=40$ (LAT2, MCT8). ATC: $n=5$ (LAT4) or $n=40$ (LAT2, MCT8). Results were considered significant if $* * P<0.01$ and $* * * P<0.001$, Olympus BX51 upright microscope (scale bar: $20 \mu \mathrm{m}$, Olympus). One-way ANOVA with Bonferroni's multiple comparison post-hoc test. Representative examples are shown. 
Table 1 Immunohistochemical analysis of MCT8, LAT2 and LAT4 in human tissue of NT, FA, FTC, PTC and ATC.

\begin{tabular}{|c|c|c|c|}
\hline TH transporter & Thyroid entity & Number & Mean H-score \pm S.E.M. \\
\hline \multirow[t]{5}{*}{ МСТ8 } & NT & 19 & $53.9 \pm 7.8$ \\
\hline & FA & 44 & $49.6 \pm 8.5$ \\
\hline & FTC & 45 & $18.6 \pm 5.8$ \\
\hline & PTC & 40 & $22.6 \pm 7.5$ \\
\hline & ATC & 40 & $3.3 \pm 2.0$ \\
\hline \multirow[t]{5}{*}{ LAT2 } & NT & 19 & $55.8 \pm 9.7$ \\
\hline & FA & 19 & $68.7 \pm 7.8$ \\
\hline & FTC & 45 & $73.3 \pm 4.3$ \\
\hline & PTC & 40 & $98.6 \pm 6.7$ \\
\hline & ATC & 40 & $39.1 \pm 5.4$ \\
\hline \multirow{5}{*}{ LAT4 } & NT & 5 & $106.3 \pm 41.6$ \\
\hline & FA & 5 & $52.0 \pm 38.1$ \\
\hline & FTC & 5 & $22.0 \pm 19.5$ \\
\hline & PTC & 5 & $11.0 \pm 3.7$ \\
\hline & ATC & 5 & $0 \pm 0$ \\
\hline
\end{tabular}

ATC, anaplastic thyroid carcinoma; FA, follicular thyroid adenoma; FTC, follicular thyroid carcinoma; LAT2, L-type amino acid transporter type 2; LAT4, L-type amino acid transporter type 4; MCT8, monocarboxylate transporter 8 ; NT, normal thyroid tissue;

PTC, papillary thyroid carcinoma.

immunofluorescence and immunocytochemistry. By immunofluorescence, MCT8 was mainly located at the plasma membrane (Fig. 3A, arrows). Immunohistochemistry of a hanging drop culture of PCCL3 cells revealed exclusive membrane staining of MCT8 in PCCL3 cells (Fig. 3B).

Since hyperthyroidism, i.e. due to GD involves augmentation of cyclic adenosine monophosphate (cAMP) signalling, we asked whether TSH stimulation impacts TH transporter expression in PCCL3 cells. MCT8 protein expression was significantly upregulated after $8 \mathrm{~h}$ of TSH stimulation (Fig. 4A).

Notably investigation of LAT2 and LAT4 expression in PCCL3 cells under TSH stimulation demonstrated that

Table 2 Immunohistochemical analysis of MCT8, LAT2 and LAT4 in human tissue of NT and GD.

\begin{tabular}{|c|c|c|c|}
\hline TH transporter & Thyroid entity & Number & Mean H-score \pm S.E.M. \\
\hline \multirow[t]{2}{*}{ МСТ8 } & NT & 19 & $53.9 \pm 7.8$ \\
\hline & GD & 50 & $126.6 \pm 6.5$ \\
\hline \multirow[t]{2}{*}{ LAT2 } & NT & 19 & $55.8 \pm 9.7$ \\
\hline & GD & 50 & $143.5 \pm 7.7$ \\
\hline \multirow[t]{2}{*}{ LAT4 } & NT & 5 & $106.3 \pm 41.6$ \\
\hline & GD & 5 & $210.0 \pm 10.0$ \\
\hline
\end{tabular}

GD, Graves' disease tissue; LAT2, L-type amino acid transporter type 2; LAT4, L-type amino acid transporter type 4; MCT8, monocarboxylate transporter 8; NT, normal thyroid tissue.

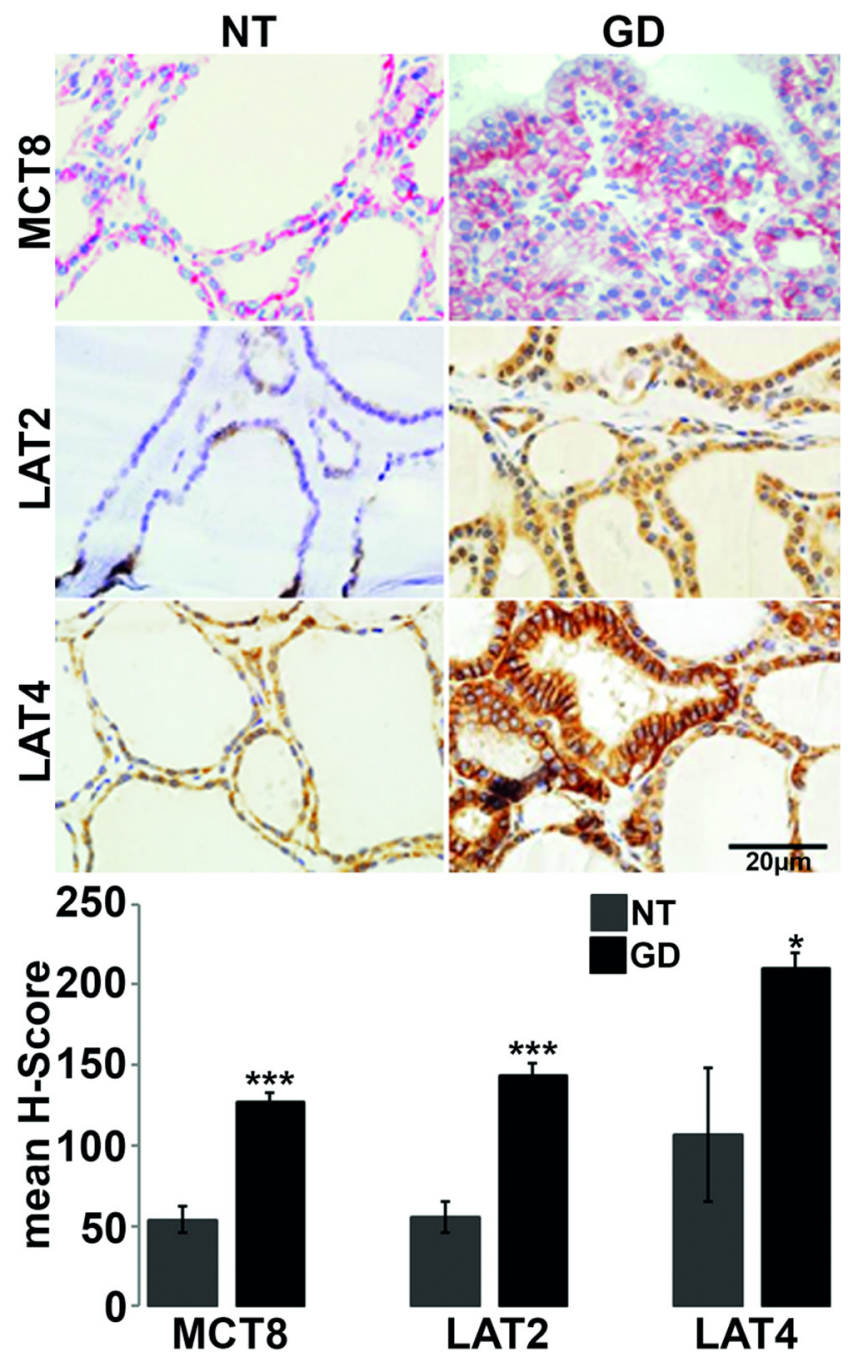

Figure 2

TH transporter expression is elevated in hyperfunctional Graves' disease tissues. Immunohistochemical analysis of TH transporter expression in normal thyroid tissues (NT) and Graves' disease (GD). Significant upregulation of monocarboxylate transporter 8 (MCT8), L-type amino acid transporter type 2 (LAT2) and LAT4 was found in GD as compared to NT. MCT8 and LAT4 show plasma membrane localization. Expression levels in GD were normalized to NT. Data are represented as mean H-Scores, mean \pm S.E.M. NT: $n=5$ (LAT4) or $n=19$ (MCT8, LAT2). GD: $n=5$ (LAT4) or $n=50$ (MCT8, LAT2). Results were considered significant if $* P<0.05$, $* * * P<0.001$, one-way ANOVA with Bonferroni's multiple comparison post-hoc test. Olympus BX51 upright microscope (scale bar: $20 \mu \mathrm{m}$, Olympus). Representative examples are shown. 
A
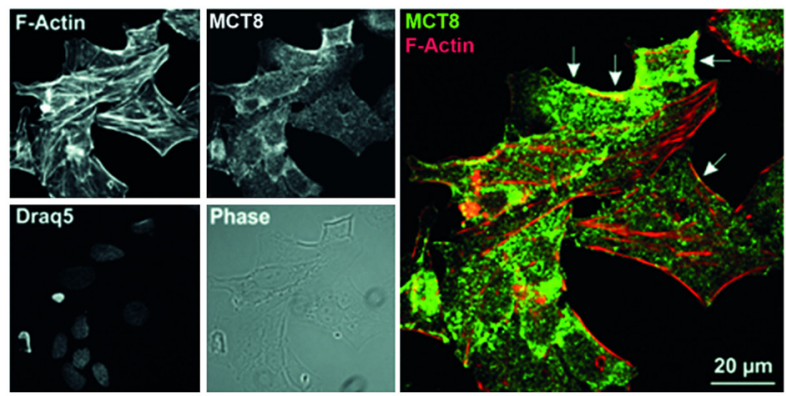

B

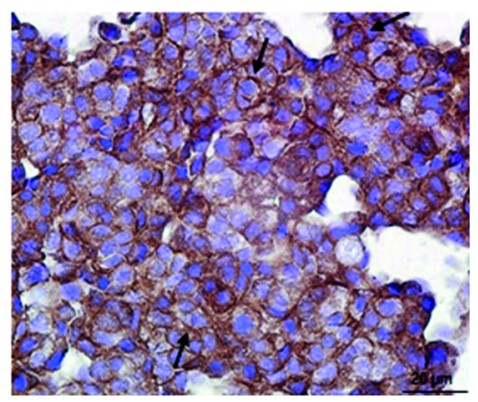

Figure 3

MCT8 is mainly located at the plasma membrane of PCCL3 cells. (A) PCCL3 cells with predominant monocarboxylate transporter 8 (MCT8, green) localization at the plasma membrane (highlighted by arrows) by immuno-fluorescence. Detection of nuclear DNA was performed with Draq5 (blue), while the F-actin cytoskeleton was visualized by Alexa 555-coupled Phalloidin staining (red). Microscopy was performed with confocal microscopy using the LSM510 (Zeiss) Merged and single channel images are depicted as indicated. (B) Immunocytochemical analysis of MCT8 in PCCL3 cells. MCT8 is located in the plasma membrane of PCCL3 cells. Olympus BX51 upright microscope (60x, Olympus). Scale bar: $20 \mu \mathrm{m}, n=3$. Representative examples are shown.

neither LAT2 nor LAT4 expression levels were altered by TSH stimulation of PCCL3 cells (Fig. 4B and C). These in vitro data suggest TSH-dependent regulation of MCT8 but no other investigated transporters in thyroid tissues.

\section{Discussion}

In this study, we asked whether $\mathrm{TH}$ transporter expression in human thyroid tissues is linked to morphology and functional tissue status, which to our knowledge has not been investigated in detail so far. For immunohistochemistry we employed a large series of 238 thyroid tissues, comprising NTs, FA, FTC, PTC, ATC and GD tissues and used a semi-quantitative scoring system to convert expert, but still subjective perception of protein expression into quantitative data which can be used for statistical analysis. The H-Score scoring system is a reproducible scoring system which is applied by pathologists for routine immuno-analysis of human tissue specimen (13).

First, we addressed MCT8 since it is the most specific and best investigated $\mathrm{TH}$ transporter. By immunohistochemistry, we found moderate plasma membrane staining for MCT8 in human thyroid tissue samples. Furthermore we observed downregulation of MCT8 in thyroid cancers (FTC, PTC and ATC) compared to NT. Since MCT8 has been shown to play a role in TH export from thyroid follicles, this is in line with a decrease in efficient TH synthesis as known for thyroid cancers (15). Hence MCT8 could represent a suitable marker of thyroid differentiation. A potential role for MCT8 and alterations of TH intra-thyroidal tissue states has been discussed on the basis of a case report of PTC diagnosed in a patient with MCT8 deficiency and the finding of papillary thyroid structures in the Mct8 knock-out mouse model at 600 day of age (16). Studies using mice with a global deficiency in MCT8, and those doubly deficient in MCT8 and the related MCT10, revealed a molecular mechanistic explanation for such auto-thyrotoxic states (6). Upon altered TH transport capabilities of thyrocytes due to lacking MCT8 and/or MCT10, thyroglobulin storage is affected and enhanced utilization of thyroglobulin for $\mathrm{TH}$ liberation is observed (6), thereby explaining the increased intra-thyroidal $\mathrm{TH}$ levels (17). The pivotal role of MCT8 in TH synthesis and release is also illustrated in our analysis of GD specimen showing markedly increased MCT8 transporter expression, consistent with a functional role of MCT8 in augmented $\mathrm{TH}$ release in hyperthyroidism. One shortcoming of this study is that clinical data of our patients are lacking. On the other hand, we clearly show a regulation of MCT8 expression in thyroid tumours and hyperfunctioning tissues of GD. Furthermore, MCT8 expression is upregulated by TSH stimulation shown in our in vitro experiments in PCCL3 cells suggesting a cAMP dependent upregulation of MCT8 consistent with other proteins relevant for efficient TH synthesis including the sodium iodide symporter (NIS) (18). We also addressed the localization pattern of MCT8 in the rat thyroid cell line PCCL3. Results are in agreement with immunohistochemical studies of human tissue samples in that MCT8 is mainly located at the cell surface between neighbouring cells, i.e. at the basolateral plasma membrane domain of thyrocytes.

Interestingly, a similar regulation pattern as for MCT8 was found for the TH transporter LAT4 both in human ATC and hyperfunctional thyroid tissues in vitro and in vivo. Although previous studies suggest that LAT4 does 
A

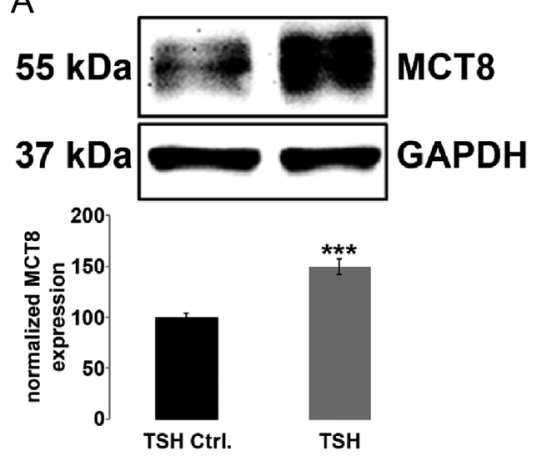

B

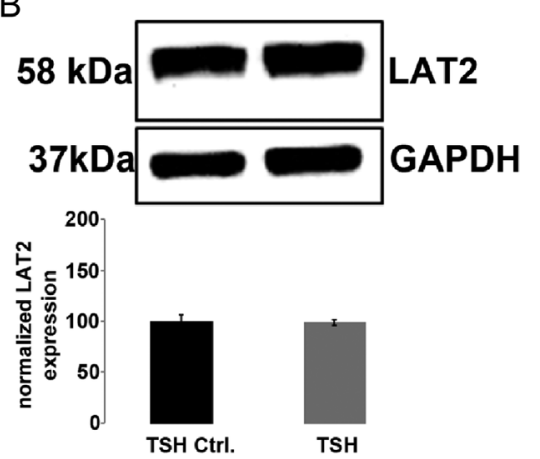

C

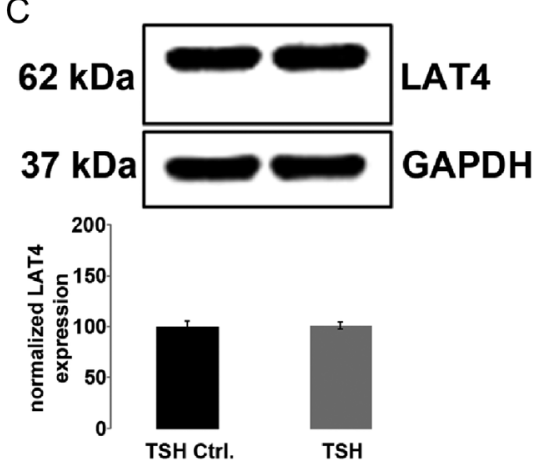

Figure 4

MCT8 expression is regulated by TSH stimulation in PCCL3 cells. Immunoblot analysis of PCCL3 cells after stimulation with thyroid stimulating hormone $\left(\mathrm{TSH}, 1 \mathrm{U} / \mathrm{mL}\right.$ ) for $8 \mathrm{~h}$ at $37^{\circ} \mathrm{C}$. (A) Significant upregulation of monocarboxylate transporter 8 (MCT8) after TSH stimulation. (B) No differences in L-type amino acid transporter type 2 (LAT2) expression by TSH stimulation of PCCL3 cells. (C) No differences in LAT4 expression by TSH stimulation of PCCL3 cells. Representative examples are shown. Data are represented as mean \pm S.E.M. of three independent experiments, Student's $t$-test, $* * * P<0.001$.

not function as a transporter of classical TH T3 and T4 (10), LAT4 expression patterns in GD tissues strongly suggest that this $\mathrm{TH}$ transporter contributes to adaptation of thyroidal $\mathrm{TH}$ homeostasis in hyperthyroidism if not exerting a functional role in $\mathrm{TH}$ release. In contrast to TSH-dependent upregulation of MCT8 in PCCL3 cells, LAT4 protein level was not altered by TSH. Therefore, LAT4 downregulation in ATC as well as LAT4 upregulation in GD tissues might hint to other functional roles of this transporter like amino acid rather than TH transport.

For LAT2 immunostaining of human thyroid specimen revealed a heterogeneous expression pattern. Thus, for LAT2 we found moderate staining mainly in the cytoplasm. Normally, plasma membrane localization would be expected for $\mathrm{TH}$ transporters exporting $\mathrm{TH}$ from thyrocytes $(19,20)$. However, in our series LAT2 was mainly located in cytoplasmic structures, presumably in vesicles of the endocytic pathway, which might be linked to the hypothesis of an endo-lysosomal functioning protein of LAT2 in the thyroid. Quantification of immunohistochemical analysis showed a significant upregulation of LAT2 in PTC compared to NT. Likewise, in GD, we found significant upregulation for LAT2. Thus, expression pattern of LAT2 observed in the different tissues are more likely reflecting other transporter properties of LAT2, i.e., to maintain influx of essential amino acids. In accordance, TSH stimulation of PCCL3 cells did not affect LAT2 expression.

In summary, we observed a gradual downregulation of MCT8 in FA and thyroid carcinomas (FTC, PTC and ATC) which would be in agreement with the activation of TK signalling pathways over cAMP dependent maintenance of thyroid cell differentiation during thyroid carcinogenesis. Additionally, upregulation of the TH transporter MCT8 in hyperfunctioning GD tissues is TSH-dependent and consistent with the proposed role in TH release.

In conclusion, MCT8 represents as a novel thyroid differentiation marker and could be involved in excessive TH supply in states of hyperthyroidism, thereby contributing to disease manifestation.

\section{Declaration of interest}

The authors declare that there is no conflict of interest that could be perceived as prejudicing the impartiality of this study.

\section{Funding}

This study was supported by grants by the Deutsche Forschungsgemeinschaft (DFG) within the SPP1629 framework to D F (FU356/7-1), D Z (ZW221/2-1), K B (BR1308/11-1), L M (MO1018/2-1) and by the Deutsche Krebshilfe to G B (\#106294).

\section{Acknowledgements}

The authors thank Pilar Santisteban (Instituto de Investigaciones Biomédicas 'Alberto Sols' CSIC-UAM, Madrid, Spain) for providing the PCCL3 cells. They thank the Imaging Core Facility Essen (IMCES) for its support with the Olympus microscope. They are also grateful to S Rehn, A Jaeger and M Rehders for their dedicated technical support.

\section{References}

1 Yen PM. Physiological and molecular basis of thyroid hormone action. Physiological Reviews 200181 1097-1142.

2 Friesema EC, Ganguly S, Abdalla A, Manning Fox JE, Halestrap AP \& Visser TJ. Identification of monocarboxylate transporter 8 as a specific thyroid hormone transporter. Journal of Biological Chemistry 2003278 40128-40135. (doi:10.1074/jbc.M300909200) 
3 Di Cosmo C, Liao XH, Dumitrescu AM, Philp NJ, Weiss RE \& Refetoff S. Mice deficient in MCT8 reveal a mechanism regulating thyroid hormone secretion. Journal of Clinical Investigation $2010 \mathbf{1 2 0}$ 3377-3388. (doi:10.1172/JCI42113)

4 Kinne A, Schulein R \& Krause G. Primary and secondary thyroid hormone transporters. Thyroid Research 20114 (Supplement 1) S7. (doi:10.1186/1756-6614-4-S1-S7)

5 Chan SY, Franklyn JA, Pemberton HN, Bulmer JN, Visser TJ, McCabe CJ \& Kilby MD. Monocarboxylate transporter 8 expression in the human placenta: the effects of severe intrauterine growth restriction. Journal of Endocrinology 2006189 465-471. (doi:10.1677/joe.1.06582)

6 Weber J, McInnes J, Kizilirmak C, Rehders M, Qatato M, Wirth EK, Schweizer U, Verrey F, Heuer H \& Brix K. Interdependence of thyroglobulin processing and thyroid hormone export in the mouse thyroid gland. European Journal of Cell Biology 2017 [in press]. (doi:10.1016/j.ejcb.2017.02.002)

7 Friesema EC, Kuiper GG, Jansen J, Visser TJ \& Kester MH. Thyroid hormone transport by the human monocarboxylate transporter 8 and its rate-limiting role in intracellular metabolism. Molecular Endocrinology 200620 2761-2772. (doi:10.1210/me.2005-0256)

8 Wagner CA, Lang F \& Broer S. Function and structure of heterodimeric amino acid transporters. American Journal of Physiology: Cell Physiology 2001281 C1077-C1093.

9 Kinne A, Wittner M, Wirth EK, Hinz KM, Schulein R, Kohrle J \& Krause G. Involvement of the L-type amino acid transporter Lat2 in the transport of 3,3'-diiodothyronine across the plasma membrane. European Thyroid Journal 20154 42-50. (doi:10.1159/000381542)

10 Zevenbergen C, Meima ME, Lima de Souza EC, Peeters RP, Kinne A, Krause G, Visser WE \& Visser TJ. Transport of iodothyronines by human L-type amino acid transporters. Endocrinology 2015156 4345-4355. (doi:10.1210/en.2015-1140)

11 Pineda M, Fernandez E, Torrents D, Estevez R, Lopez C, Camps M, Lloberas J, Zorzano A \& Palacin M. Identification of a membrane protein, LAT-2, that co-expresses with 4F2 heavy chain, an L-type amino acid transport activity with broad specificity for small and large zwitterionic amino acids. Journal of Biological Chemistry 1999 274 19738-19744. (doi:10.1074/jbc.274.28.19738)

12 Bodoy S, Martin L, Zorzano A, Palacin M, Estevez R \& Bertran J. Identification of LAT4, a novel amino acid transporter with system
L activity. Journal of Biological Chemistry 2005280 12002-12011. (doi:10.1074/jbc.M408638200)

13 Ting S, Mairinger FD, Hager T, Welter S, Eberhardt WE, Wohlschlaeger J, Schmid KW \& Christoph DC. ERCC1, MLH1, MSH2, MSH6, and betaIII-tubulin: resistance proteins associated with response and outcome to platinum-based chemotherapy in malignant pleural mesothelioma. Clinical Lung Cancer 201314 558.e553-567.e553. (doi:10.1016/j.cllc.2013.04.013)

14 Fusco A, Berlingieri MT, Di Fiore PP, Portella G, Grieco M \& Vecchio G. One- and two-step transformations of rat thyroid epithelial cells by retroviral oncogenes. Molecular and Cellular Biology 19877 3365-3370. (doi:10.1128/MCB.7.9.3365)

15 Krause K, Karger S, Schierhorn A, Poncin S, Many MC \& Fuhrer D. Proteomic profiling of cold thyroid nodules. Endocrinology 2007148 1754-1763. (doi:10.1210/en.2006-0752)

16 Wirth EK, Sheu SY, Chiu-Ugalde J, Sapin R, Klein MO, Mossbrugger I, Quintanilla-Martinez L, de Angelis MH, Krude H, Riebel T et al. Monocarboxylate transporter 8 deficiency: altered thyroid morphology and persistent high triiodothyronine/thyroxine ratio after thyroidectomy. European Journal of Endocrinology 2011165 555-561. (doi:10.1530/EJE-11-0369)

17 Muller J \& Heuer H. Expression pattern of thyroid hormone transporters in the postnatal mouse brain. Frontiers in Endocrinology 20145 92. (doi:10.3389/fendo.2014.00092)

18 Ohno M, Zannini M, Levy O, Carrasco N \& di Lauro R. The paireddomain transcription factor Pax8 binds to the upstream enhancer of the rat sodium/iodide symporter gene and participates in both thyroid-specific and cyclic-AMP-dependent transcription. Molecular and Cellular Biology 199919 2051-2060. (doi:10.1128/МСB.19.3.2051)

19 McInnes J, Weber J, Rehders M, Saftig P, Peters C, Reinheckel T, Schweizer U, Heuer H, Wirth EK \& Brix K. Correlation of the expression and localization of thyroid hormone transporters with thyroglobulin procesing cathepsins in mouse thyroid epithelial cells. In 37th Annual Meeting of the European Thyroid Association, pp 75-194. Leiden, The Netherlands, 2013.

20 Weber J, Rehders M, Saftig P, Verrey F, Schweizer U, Wirth EK, Heuer $\mathrm{H} \&$ Brix K. Functional analysis of the angio-follicular unit of the mouse thyroid gland. In Experimental and Clinical Endocrinology and Diabetes, pp P12-P13; 2015.

Received 6 April 2017

Revised version received 8 May 2017

Accepted 2 June 2017 